\title{
Amides, Isoquinoline Alkaloids and Dipeptides from the Aerial Parts of Piper mullesua
}

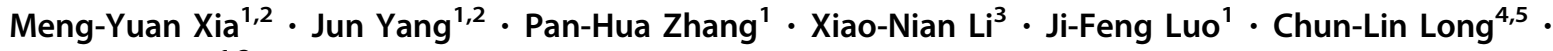 \\ Yue-Hu Wang ${ }^{1,2}$
}

Received: 3 June 2018 / Accepted: 3 July 2018 / Published online: 2 August 2018

(C) The Author(s) 2018

\section{Abstract}

One undescribed amide, pipermullesine A, two undescribed isoquinoline alkaloids, pipermullesines B and C, and six undescribed dipeptides, pipermullamides A-F, along with 28 known compounds, were isolated from the aerial parts of Piper mullesua. The structures of the undescribed compounds were elucidated based on the analysis of 1D and 2D NMR and MS data. Furthermore, the structures of pipermullesines A-C were confirmed by single crystal X-ray diffraction analysis. All isolates were evaluated for inhibitory activity against platelet aggregation induced by thrombin (IIa) or platelet-activating factor (PAF). (-)-Mangochinine, pellitorine, and $(2 E, 4 E)$ - $N$-isobutyl-2,4-dodecadienamide showed weak inhibitory activity against rabbit platelet aggregation induced by PAF, with $\mathrm{IC}_{50}$ values of $470.3 \mu \mathrm{g} / \mathrm{mL}, 614.9 \mu \mathrm{g} / \mathrm{mL}$, and $579.7 \mu \mathrm{g} / \mathrm{mL}$, respectively.

\section{Graphical Abstract}

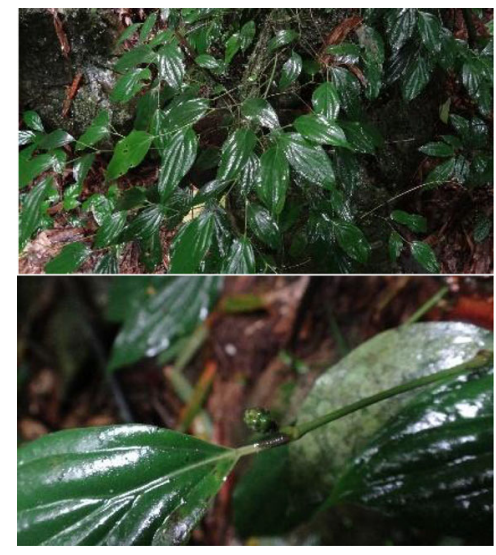<smiles>CC(=O)NCCCCC1=NCCc2cc(O)c3oc(CCCNC(C)=O)nc3c21</smiles>

pipermullesine $\mathrm{C}$<smiles>COc1cc2c(cc1O)C[C@@H]1c3cc(O)c(OC)cc3N(C)CC[C@@H]21</smiles>

(-)-mangochinine

Meng-Yuan Xia and Jun Yang have contributed equally to this work.

Electronic supplementary material The online version of this article (https://doi.org/10.1007/s13659-018-0180-z) contains supplementary material, which is available to authorized users.

Chun-Lin Long

long@mail.kib.ac.cn

Yue-Hu Wang

wangyuehu@mail.kib.ac.cn

1 Key Laboratory of Economic Plants and Biotechnology and the Yunnan Key Laboratory for Wild Plant Resources, Kunming Institute of Botany, Chinese Academy of Sciences, Kunming 650201, People's Republic of China<smiles>CCCCC/C=C/C=C/C(=O)NCC(C)C</smiles>

Southeast Asia Biodiversity Research Institute, Chinese Academy of Sciences, Yezin, Nay Pyi Taw 05282, Myanmar

3 State Key Laboratory of Phytochemistry and Plant Resources in West China, Kunming Institute of Botany, Chinese Academy of Sciences, Kunming 650201, People's Republic of China

4 College of Life and Environmental Sciences, Minzu University of China, Beijing 100081, People's Republic of China 
Keywords Piper mullesua $\cdot$ Piperaceae $\cdot$ Antiplatelet $\cdot$ Amides $\cdot$ Isoquinoline alkaloids

\section{Introduction}

Traditional Chinese medicines with the functions of promoting blood circulation ("Huoxue" in Chinese) and/ or removing blood stasis ("Huayu" in Chinese) are claimed to be useful in antiplatelet therapies and the treatment of thrombotic diseases [1]. For example, antiplatelet compounds have been found in a Huoxue herb Selaginella moellendorffii Hieron. (Selaginellaceae) $[2,3]$.

The genus Piper (Piperaceae) is a medicinally important group of plants consisting of approximately 2000 species worldwide. There are approximately 60 species distributed in the tropical areas of the People's Republic of China, of which approximately 30 species have been used as traditional Chinese medicines [4]. Some Piper species are used for promoting blood circulation, while Piper mullesua Buch.-Ham. ex D. Don and $P$. yunnanense Tseng are used for removing blood stasis [5]. As a folk medicine in China with the Chinese name of Duan-Jv (短蒟), the whole plants of $P$. mullesua are also used to treat bleeding, bone fractures, injuries from falls, rheumatoid arthritis, rheumatic arthralgia, acroanesthesia, asthma, colds, stomach aches, abdominal pain, toothaches, swelling and pain of furuncles, dysmenorrhea, menoxenia, empyrosis, and snake and insect bites $[5,6]$.

Alcoholic extracts of $P$. mullesua showed the activity against rabbit platelet aggregation induced by $7.2 \mathrm{nM}$ of the platelet-activating factor (PAF) with an $\mathrm{IC}_{50}$ value of $64.43 \mu \mathrm{g} / \mathrm{mL}$ [7]. Amides including retrofractamide A, chingchengenamide A [6], $N$-isobutyl-16-phenylhexadeca-2E,4E-dienamide, and $N$-isobutyldeca- $2 E, 4 E$-dienamide [8], lignans including (-)-nectandrin $\mathrm{A}$, nectandrin B, galgravin [6], asarinin, fargesin, and sesamin with antifeedant activity [8,9], a phenylpropanoid myristicin with insecticidal activity $[9,10]$, and several arylalkenyl carboxylic acid esters $[10,11]$ have been isolated from the plants. However, the active constituents of $P$. mullesua responsible for the antiplatelet aggregation remain unclear. In continuing efforts to search for antiplatelet compounds from Piper plants [12, 13], we herein present the results of the analysis of compounds from the aerial parts of $P$. mullesua and the bioactivity of these compounds.

\section{Results and Discussion}

\subsection{Structure Elucidation}

Nine undescribed compounds (1-9, Fig. 1) and 28 known ones (10-37) were isolated from the methanolic extracts of P. mullesua by silica gel, D101 resin and Sephadex LH-20 column chromatography and semipreparative HPLC.

Pipermullesine A (1) had the molecular formula $\mathrm{C}_{15} \mathrm{H}_{15} \mathrm{NO}_{4}$ based on ${ }^{13} \mathrm{C}$ NMR (Table 1) and HREIMS data. Its IR spectrum showed absorption peaks for a tertiary amide at $1643 \mathrm{~cm}^{-1}$ and a phenyl ring at 1595,1513 , and $1461 \mathrm{~cm}^{-1}$. The ${ }^{1} \mathrm{H}$ NMR data (Table 1) indicated a 1,2,4trisubstituted phenyl ring $\left[\delta_{\mathrm{H}} 7.12(1 \mathrm{H}, \mathrm{dd}, J=8.3,1.8 \mathrm{~Hz})\right.$, $7.01(1 \mathrm{H}, \mathrm{d}, J=1.8 \mathrm{~Hz})$, and $6.86(1 \mathrm{H}, \mathrm{d}, J=8.3 \mathrm{~Hz})]$, an $E$ double bond $\left[\delta_{\mathrm{H}} 7.67(1 \mathrm{H}, \mathrm{d}, J=15.3,1.8 \mathrm{~Hz})\right.$ and 6.49 $(1 \mathrm{H}, \mathrm{d}, J=15.3 \mathrm{~Hz})]$, a 1,4 -oxazine ring $\left[\delta_{\mathrm{H}} 6.61(1 \mathrm{H}, \mathrm{dd}, J\right.$ $=5.1,1.9 \mathrm{~Hz}), 6.10(1 \mathrm{H}, \mathrm{dd} J=5.1,1.9 \mathrm{~Hz}), 5.84(1 \mathrm{H}, \mathrm{d}, J=$ $5.1 \mathrm{~Hz})$, and $5.70(1 \mathrm{H}, \mathrm{d}, J=5.1 \mathrm{~Hz})]$ [14], and two methoxy groups $\left[\delta_{\mathrm{H}} 3.92(3 \mathrm{H}, \mathrm{s})\right.$ and $\left.3.91(3 \mathrm{H}, \mathrm{s})\right]$. The above NMR characteristic signals implied that compound $\mathbf{1}$ might be a cinnamamide derivative.

According to the ${ }^{1} \mathrm{H}-{ }^{1} \mathrm{H}$ COSY and HMBC correlations of compound 1 (Fig. 2), (E)-3,4-dimethoxycinnamoyl and 1,4-oxazine groups were confirmed. Although the correlations from $\mathrm{H}-1$ " and $\mathrm{H}-4$ " to $\mathrm{C}-1$ were not observed in the HMBC spectrum, the structure of $\mathbf{1}$ was finally determined as (E)-3-(3,4-dimethoxyphenyl)-1-(4H-1,4-oxazin-4-yl) prop-2-en-1-one by a single-crystal $\mathrm{X}$-ray diffraction analysis (Fig. 3).

The molecular formula of pipermullesine $\mathrm{B}$ (2), $\mathrm{C}_{16} \mathrm{H}_{22} \mathrm{~N}_{2} \mathrm{O}_{3}$, was determined by ${ }^{13} \mathrm{C}$ NMR data (Table 2) and an HREIMS ion at $\mathrm{m} / \mathrm{z}, 290.1620 \mathrm{CM}^{+}$(calcd for $\mathrm{C}_{16} \mathrm{H}_{22} \mathrm{~N}_{2} \mathrm{O}_{3}, 290.1630$ ) and required 7 indices of hydrogen deficiency. The ${ }^{1} \mathrm{H}$ NMR data (Table 2) indicated a tetrasubstituted phenyl ring $\left[\delta_{\mathrm{H}} 7.15(1 \mathrm{H}, \mathrm{s})\right.$ and $\left.6.52(1 \mathrm{H}, \mathrm{s})\right]$, one methoxy group $\left[\delta_{\mathrm{H}} 3.85(3 \mathrm{H}, \mathrm{s})\right]$, and one acetyl group $\left[\delta_{\mathrm{H}} 1.91(3 \mathrm{H}, \mathrm{s})\right]$. The ${ }^{13} \mathrm{C}$ NMR data (Table 2) exhibited 15 signals. However, according to its HREIMS data, compound 2 should have 16 carbon atoms. The disappeared signal for C-9 $\left(\delta_{\mathrm{C}} 33.0\right)$ was detected by the HMBC correlation (Fig. 2) from $\mathrm{H}_{2}-11$ to C-9.

${ }^{1} \mathrm{H}-{ }^{1} \mathrm{H}$ COSY correlations (Fig. 2) exhibited two partial structures comprising $\mathrm{C}-2$ to $\mathrm{C}-3$ and $\mathrm{C}-10$ to $\mathrm{C}-12$. On the basis of the HMBC correlations from $\mathrm{H}_{2}-3$ to $\mathrm{C}-1$ and C- $4 \mathrm{a}, \mathrm{H}_{2}-4$ to C-5 and C-8a, H-5 to C-7 and C-8a, H-8 to $\mathrm{C}-1$, C-4a, and C-6, and 7-OMe to C-7, a 6-hydroxy-7methoxy-3,4-dihydroisoquinoline fragment with a substituent group at $\mathrm{C}-1$ was confirmed. The group at $\mathrm{C}-1$ was deduced as 4-acetamidobutyl by the HMBC correlations 
<smiles>COc1ccc(/C=C/C(=O)N2C=COC=C2)cc1OC</smiles><smiles>COc1cc2c(cc1O)CCN=C2CCCCNC(C)=O</smiles><smiles></smiles><smiles></smiles><smiles>CC(C)[C@H](C(=O)N[C@@H](Cc1ccccc1)C(=O)[O-])[N+](C)(C)C</smiles><smiles>CC(=O)NCCCCC1=NCCc2cc(O)c3oc(CCCCNC(C)=O)nc3c21</smiles><smiles>CC(C)CC(C(=O)NC(Cc1c[nH]c2ccccc12)C(=O)[O-])N(C)C</smiles><smiles>CC[C@H](C)[C@H](C(=O)N[C@H](Cc1c[nH]c2ccccc12)C(=O)[O-])[N+](C)(C)C</smiles>

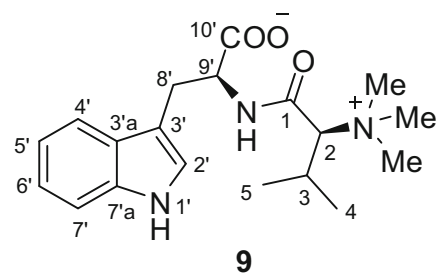

Fig. 1 Structures of undescribed compounds (1-9) from Piper mullesua

Table $1{ }^{1} \mathrm{H}(500 \mathrm{MHz})$ and ${ }^{13} \mathrm{C}$ NMR (125 MHz) NMR Data of 1 in $\mathrm{CDCl}_{3}$

\begin{tabular}{lll}
\hline No. & $\delta_{\mathrm{H}}(\mathrm{J}$ in Hz) & $\delta_{\mathrm{C}}$, type \\
\hline 1 & & $158.3, \mathrm{C}$ \\
2 & $6.49, \mathrm{~d}(15.3)$ & $112.0, \mathrm{CH}$ \\
3 & $7.67, \mathrm{~d}(15.3)$ & $144.4, \mathrm{CH}$ \\
$1^{\prime}$ & & $128.0, \mathrm{C}$ \\
$2^{\prime}$ & $7.01, \mathrm{~d}(1.8)$ & $110.0, \mathrm{CH}$ \\
$3^{\prime}$ & & $149.3, \mathrm{C}$ \\
$4^{\prime}$ & & $151.1, \mathrm{C}$ \\
$5^{\prime}$ & $6.86, \mathrm{~d}(8.3)$ & $111.2, \mathrm{CH}$ \\
$6^{\prime}$ & $7.12, \mathrm{dd}(8.3,1.8)$ & $122.4, \mathrm{CH}$ \\
$1^{\prime \prime}$ & $6.61, \mathrm{dd}(5.1,1.9)$ & $108.7, \mathrm{CH}$ \\
$2^{\prime \prime}$ & $5.84, \mathrm{~d}(5.1)$ & $132.7, \mathrm{CH}$ \\
$3^{\prime \prime}$ & $5.70, \mathrm{~d}(5.1)$ & $130.5, \mathrm{CH}$ \\
$4^{\prime \prime}$ & $6.10, \mathrm{dd}(5.1,1.9)$ & $109.4, \mathrm{CH}$ \\
$3^{\prime}-\mathrm{OMe}$ & $3.92, \mathrm{~s}$ & $56.1, \mathrm{CH}$ \\
$4^{\prime}-\mathrm{OMe}$ & $3.91, \mathrm{~s}$ & $56.1, \mathrm{CH}$ \\
\hline
\end{tabular}

from $\mathrm{H}_{2}-10$ to $\mathrm{C}-1, \mathrm{H}_{2}-11$ to $\mathrm{C}-9$ and $\mathrm{C}-1^{\prime}$, and $\mathrm{H}_{3}-2^{\prime}$ to C-1'. Thus, the structure of 2 was determined as 1-(4-acetamidobutyl)-6-hydroxy-7-methoxy-3,4-dihydroisoquinoline and given the common name pipermullesine $\mathrm{B}$.

The crystals for pipermullesine B trifluoroacetate (2a) were obtained from methanol. The NMR data of $\mathbf{2 a}$ (Table 2) and the result of its single-crystal X-ray diffraction analysis (Fig. 3) further supported the structure elucidation of $\mathbf{2}$.

Pipermullesine C (3) yielded a molecular formula of $\mathrm{C}_{22} \mathrm{H}_{30} \mathrm{~N}_{4} \mathrm{O}_{4}$ with 10 degrees of unsaturation, as deduced by ${ }^{13} \mathrm{C}$ NMR (Table 3 ) and the HREIMS data. A comparison of the NMR data (Tables 2, 3) of $\mathbf{3}$ with those of $\mathbf{2}$ indicated that there were signals for one additional 4-acetamidobutyl group $\left[\delta_{\mathrm{C}} 173.2(\mathrm{C}), 40.1\left(\mathrm{CH}_{2}\right), 29.7\left(\mathrm{CH}_{2}\right)\right.$, $28.9\left(\mathrm{CH}_{2}\right), 25.2\left(\mathrm{CH}_{2}\right)$, and $\left.22.6\left(\mathrm{CH}_{3}\right)\right]$ and one more imine $\left(\delta_{\mathrm{C}} 168.8\right)$ in 3 .

On the basis of 2D NMR correlations (Fig. 2), a 1-(4acetamidobutyl)-6-hydroxy-3,4-dihydroisoquinoline moiety was determined. One more ring is needed to meet the unsaturation, and the ring was deduced as an oxazole ring attached to C-7 and C-8 by comparison of the NMR data with those of benzoxazoles in the literature $[15,16]$. The additional 4-acetamidobutyl group was located at $\mathrm{C}-1^{\prime \prime}$ of the oxazole ring by the HMBC correlation from $\mathrm{H}-3^{\prime \prime}$ to $\mathrm{C}-1^{\prime \prime}$. Thus, the structure of $\mathbf{3}$ (pipermullesine $\mathrm{C}$ ) was determined.

Fortunately, the crystals for pipermullesine $\mathrm{C}$ trifluoroacetate (3a) were also obtained from methanol. The NMR data of $\mathbf{3 a}$ (Table 3 ) and the result of its singlecrystal X-ray diffraction analysis (Fig. 3) confirmed the chemical structure of $\mathbf{3}$.

The molecular formula of pipermullamide $\mathrm{A}$ (4), $\mathrm{C}_{18} \mathrm{H}_{28} \mathrm{~N}_{2} \mathrm{O}_{3}$, was determined by ${ }^{13} \mathrm{C}$ NMR data (Table 4) and an HREIMS ion at 320.2102 [M] $^{+}$(calcd for 

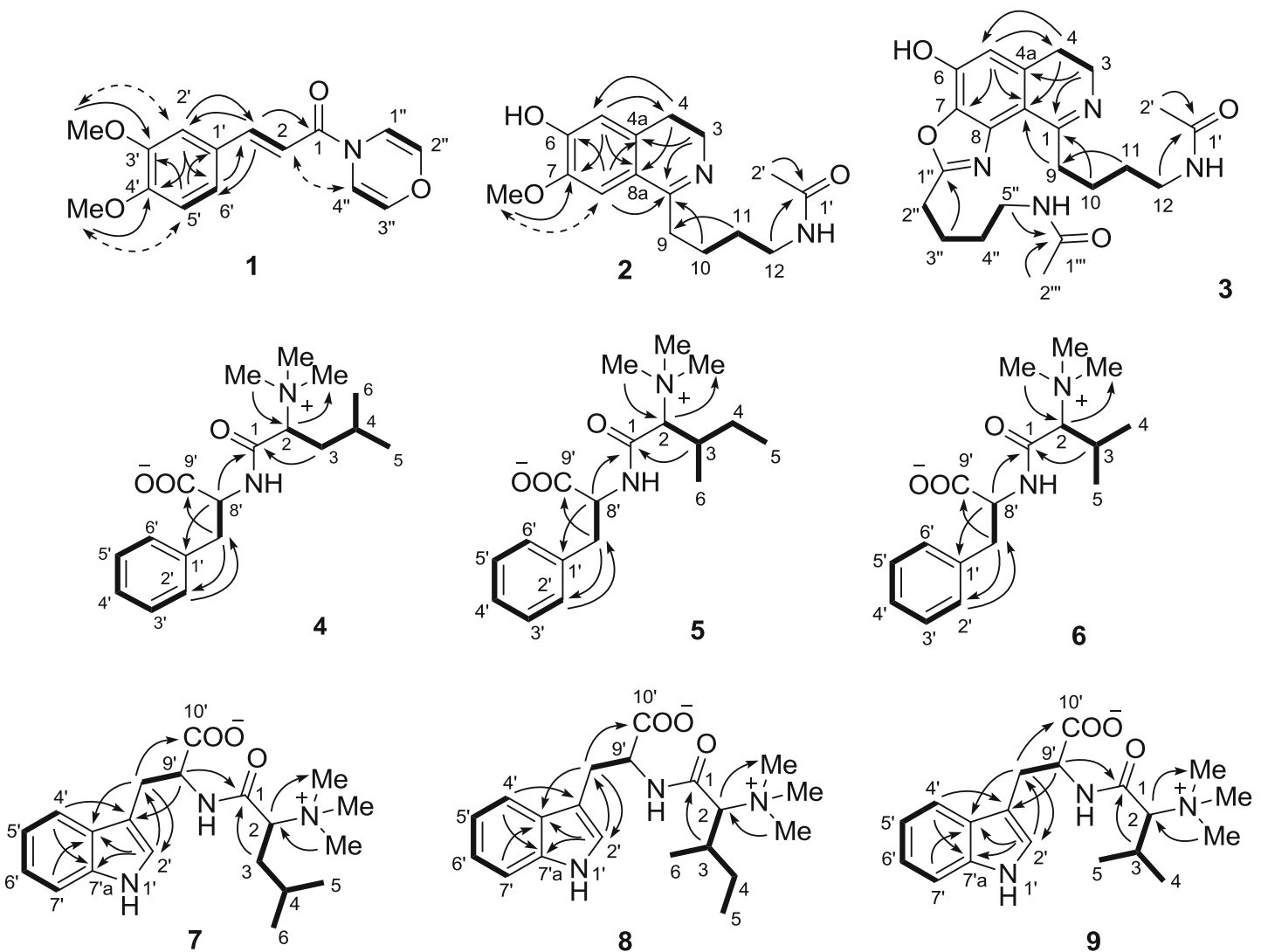

${ }^{1} \mathrm{H}-{ }^{1} \mathrm{H}$ COSY

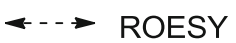

Fig. 2 Key 2D NMR correlations of compounds 1-9

$\mathrm{C}_{18} \mathrm{H}_{28} \mathrm{~N}_{2} \mathrm{O}_{3}, 320.2100$ ), indicating 6 degrees of unsaturation. The ${ }^{1} \mathrm{H}$ NMR data (Table 2) indicated one monosubstituted phenyl ring $\left[\delta_{\mathrm{H}} 7.31(2 \mathrm{H}, \mathrm{d}, J=7.4 \mathrm{~Hz}), 7.25\right.$ $(2 \mathrm{H}, \mathrm{dd}, J=7.4,7.4 \mathrm{~Hz})$, and $7.17(1 \mathrm{H}, \mathrm{dd}, J=7.4,7.4 \mathrm{~Hz})]$, three $N$-methyl groups $\left[\delta_{\mathrm{H}} 2.85(9 \mathrm{H}, \mathrm{s})\right]$, and two methyl groups $\left[\delta_{\mathrm{H}} 0.98(3 \mathrm{H}, \mathrm{d}, J=5.9 \mathrm{~Hz})\right.$ and $0.95(3 \mathrm{H}, \mathrm{d}, J=$ $6.3 \mathrm{~Hz})$ ]. By comparing the NMR data of $\mathbf{4}$ with those of phenylalanine and leucine trimethylbetaine [17, 18], compound 4 might comprise the two fragments, which was confirmed through its ${ }^{1} \mathrm{H}-{ }^{1} \mathrm{H}$ COSY and HMBC correlations (Fig. 2). The amino of phenylalanine was acylated by the carboxyl group of leucine trimethylbetaine according to the HMBC correlation from $\mathrm{H}-8^{\prime}$ to $\mathrm{C}-1$.

Natural amino acids generally have an L configuration. Compound $\mathbf{1 0}$ from the plant is also a derivative of Lphenylalanine. Accordingly, compound 4 (pipermullamide A) was elucidated as $\mathrm{L}-(N, N, N$-trimethyl)leucyl-Lphenylalanine.

The molecular formulae of pipermullamides $\mathrm{B}$ to $\mathrm{F}$ (59) were determined as $\mathrm{C}_{18} \mathrm{H}_{28} \mathrm{~N}_{2} \mathrm{O}_{3}, \quad \mathrm{C}_{17} \mathrm{H}_{26} \mathrm{~N}_{2} \mathrm{O}_{3}$, $\mathrm{C}_{20} \mathrm{H}_{29} \mathrm{~N}_{3} \mathrm{O}_{3}, \mathrm{C}_{20} \mathrm{H}_{29} \mathrm{~N}_{3} \mathrm{O}_{3}$, and $\mathrm{C}_{19} \mathrm{H}_{27} \mathrm{~N}_{3} \mathrm{O}_{3}$, respectively, by ${ }^{13} \mathrm{C}$ NMR data (Tables 4,5 ) and HRMS analysis. According to ${ }^{1} \mathrm{H}-{ }^{1} \mathrm{H}$ COSY and $\mathrm{HMBC}$ correlations
(Fig. 2), compounds 5-9 were determined as $\mathrm{L}-(N, N, N-$ trimethyl)isoleucyl-L-phenylalanine (pipermullamide B, 5), L-(N,N,N-trimethyl)valyl-L-phenylalanine (pipermullamide $\mathrm{C}, \mathbf{6})$, L-( $N, N, N$-trimethyl)leucyl-L-tryptophan (pipermullamide $\mathrm{D}, 7), \mathrm{L}-(N, N, N$-trimethyl)isoleucyl-L-tryptophan (pipermullamide $\mathrm{E}, 8)$, and $\mathrm{L}-(N, N, N$-trimethyl)valyl-Ltryptophan (pipermullamide F, 9), respectively.

The known compounds (+)-phenylalanine betaine (10) [19], (-)-mangochinine (11) [20], xylopinidine (12) [21], (-)-oblongine (13) [22], pellitorine (14) [23], $(2 E, 4 E)-N$ isobutyl-2,4-dodecadienamide (15) [24], retrofractamide A (16) [23], guineensine (17) [23], brachystamide B (18) [25], retrofractamide C (19) [26], sarmentine (20) [27], 3-(3,4-dimethoxyphenyl)propanoylpyrrole (21) [28], $\mathrm{N}$ trans-feruloyltyramine (22) [29], (-)-machilusin (23) [30], galgravin (24) [31], (-)-nectandrin A (25) [32], methyl 3-(3,4-dimethoxyphenyl)propanoate (26) [33], piperic acid (27) [34], methyl piperate (28) [34], methyl $(2 E, 4 E)-7-(1,3-$ benzodioxol-5-yl)hepta-2,4-dienoate (29) [35], (-)-blumenol B (30) [36], (-)-T-muurolol (31) [37], trans-phytol (32) [38], $\alpha$-tocopherolquinone (33) [39], $\gamma$-tocopherol (34) [40], stigmast-4-ene-3,6-dione (35) [41], (22E)-stigmasta4,22-diene-3,6-dione (36) [42], and (22E)-stigmasta-4,6,8 
<smiles>CC(C)CC1C(C)C(C)C(C(C)C(C)C(C)C2C(C)C(C)CC(C)C2C)C(C)C1CC(C)C</smiles>

1

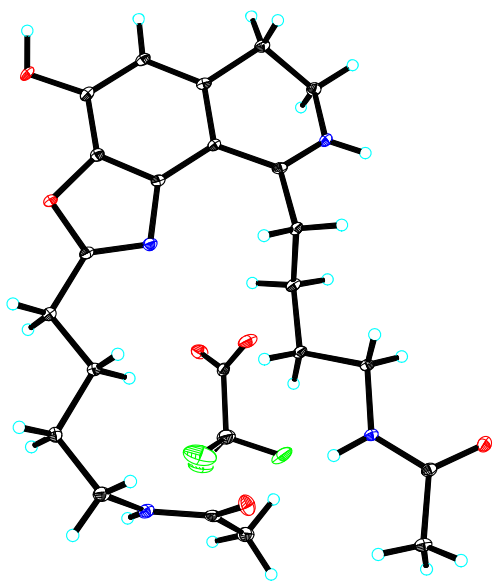

Fig. 3 X-ray crystallographic structures of $\mathbf{1}, \mathbf{2 a}$, and $\mathbf{3 a}$

(14),22-tetraen-3-one (37) [43] were determined by comparing the NMR data of 10-37 and the optical rotation values of 10-13, 23-25, 30, and $\mathbf{3 1}$ with those reported in the literature.

\subsection{In Vitro Platelet Aggregation Assay}

All isolates (1-37) were evaluated for inhibitory activity against platelet aggregation induced by thrombin (IIa) or PAF. As shown in Tables 6 and 7, compounds 2, 3, 5, 14, 27, 33, and $\mathbf{3 4}$ possessed weak inhibitory effects on the aggregation of rabbit platelets induced by thrombin (IIa) (1 $\mathrm{U} / \mathrm{mL}$ ) with inhibition rates from 11.5 to $22.2 \%$ at a concentration of $300 \mu \mathrm{g} / \mathrm{mL}$. Compounds 11, 14, 15, 20, and 25 showed weak inhibitory activity against the rabbit platelet aggregation induced by PAF $(0.4 \mu \mathrm{g} / \mathrm{mL})$ with inhibition rates from $16.8 \%$ to $36.4 \%$ at a concentration of $300 \mu \mathrm{g} / \mathrm{mL}$, while (-)-mangochinine (11), pellitorine (14), and $(2 E, 4 E)$ $\mathrm{N}$-isobutyl-2,4-dodecadienamide (15) have $\mathrm{IC}_{50}$ values of $470.3 \mu \mathrm{g} / \mathrm{mL}, 614.9 \mu \mathrm{g} / \mathrm{mL}$, and $579.7 \mu \mathrm{g} / \mathrm{mL}$, respectively. The antiplatelet activity of (-)-mangochinine and $(2 E, 4 E)-N$ isobutyl-2,4-dodecadienamide was reported for the first time. The other tested compounds were inactive.

More than fifty antiplatelet compounds, mainly including alkaloids and amides, lignans and neolignans, and

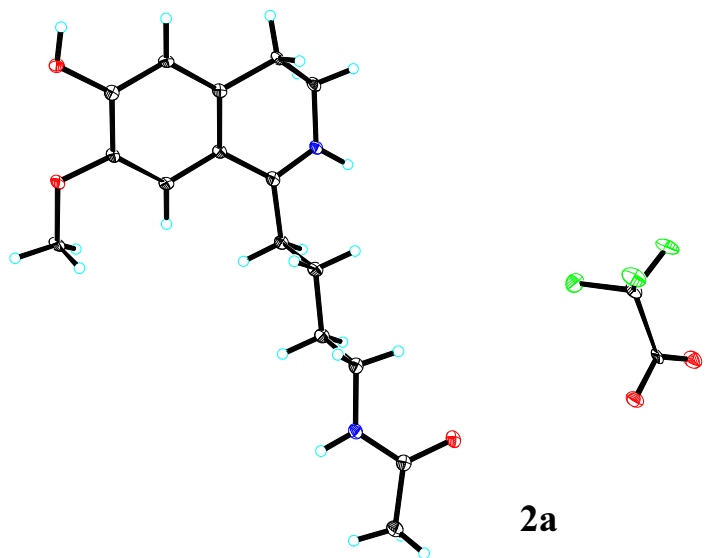

3a

phenylpropanoids, have been isolated from the Piper genus [44]. Pellitorine is a very interesting compound with various biological activities, such as an inhibitory effect on platelet aggregation induced by arachidonic acid $\left(\mathrm{IC}_{50}=\right.$ $53.0 \mu \mathrm{g} / \mathrm{mL}$ ) [45], antituberculosis activity (MIC $=25 \mu \mathrm{g} /$ $\mathrm{mL}$ ) [46], antifungal activity against Cryptococcus neoformans $\left(\mathrm{IC}_{50}=7.7 \mu \mathrm{g} / \mathrm{mL}\right)$ [47], and $\alpha$-glucosidase-I enzyme inhibitory activity $\left(\mathrm{IC}_{50}=34.39 \mu \mathrm{g} / \mathrm{mL}\right)$ [48]. Although its in vitro activity against platelet aggregation is weak, the amide shows strong in vivo anticoagulant activities at a dose of $4.5 \mu \mathrm{g} /$ mouse or $9.0 \mu \mathrm{g} /$ mouse [49]. It is worthwhile to conduct further in vivo antithrombotic studies of pellitorine along with (-)-mangochinine and (2E,4E)- $N$-isobutyl-2,4-dodecadienamide.

\section{Experimental Section}

\subsection{General Experimental Procedures}

The instruments and materials for isolation and identification of compounds from the herb were presented in Supplementary Material. 
Table $2{ }^{1} \mathrm{H}$ and ${ }^{13} \mathrm{C}$ NMR Data of $\mathbf{2}$ and $\mathbf{2 a}$ in $\mathrm{CD}_{3} \mathrm{OD}$

\begin{tabular}{|c|c|c|c|c|}
\hline \multirow[t]{2}{*}{ No. } & \multicolumn{2}{|l|}{2} & \multicolumn{2}{|l|}{$\mathbf{2 a}$} \\
\hline & $\delta_{\mathrm{H}}(J \text { in } \mathrm{Hz})^{\mathrm{a}}$ & $\delta_{C}$, type $^{b}$ & $\delta_{\mathrm{H}}(J \text { in } \mathrm{Hz})^{\mathrm{c}}$ & $\delta_{\mathrm{C}}$, type $^{\mathrm{d}}$ \\
\hline 1 & & $174.4, \mathrm{C}$ & & $179.1, \mathrm{C}$ \\
\hline 3 & $3.63, \mathrm{t}(7.7)$ & $42.4, \mathrm{CH}_{2}$ & $3.77, \mathrm{t}(7.9)$ & $42.3, \mathrm{CH}_{2}$ \\
\hline 4 & $2.87, \mathrm{t}(7.7)$ & 27.1, $\mathrm{CH}_{2}$ & $3.01, \mathrm{t}(7.9)$ & 26.0, $\mathrm{CH}_{2}$ \\
\hline $4 a$ & & 137.6, C & & $136.4, \mathrm{C}$ \\
\hline 5 & $6.52, \mathrm{~s}$ & $118.2, \mathrm{CH}$ & $6.85, \mathrm{~s}$ & $116.7, \mathrm{CH}$ \\
\hline 6 & & 167.6, C & & $158.3, \mathrm{C}$ \\
\hline 7 & & 151.1, C & & $149.2, \mathrm{C}$ \\
\hline 8 & $7.15, \mathrm{~s}$ & $112.2, \mathrm{CH}$ & $7.42, \mathrm{~s}$ & $114.1, \mathrm{CH}$ \\
\hline $8 \mathrm{a}$ & & $112.3, \mathrm{C}$ & & $116.9, \mathrm{C}$ \\
\hline 9 & Disappeared & $33.0^{\mathrm{e}}$ & Disappeared & $33.1^{\mathrm{e}}$ \\
\hline 10 & $1.72, \mathrm{~m}$ & $26.9, \mathrm{CH}_{2}$ & $1.73, \mathrm{~m}$ & $26.3, \mathrm{CH}_{2}$ \\
\hline 11 & $1.60, \mathrm{~m}$ & $29.9, \mathrm{CH}_{2}$ & $1.61, \mathrm{~m}$ & $29.9, \mathrm{CH}_{2}$ \\
\hline 12 & $3.20, \mathrm{t}(6.9)$ & $40.0, \mathrm{CH}_{2}$ & $3.20, \mathrm{t}(6.9)$ & $39.6, \mathrm{CH}_{2}$ \\
\hline $1^{\prime}$ & & 173.3, C & & $173.4, \mathrm{C}$ \\
\hline $2^{\prime}$ & $1.91, \mathrm{~s}$ & $22.6, \mathrm{CH}_{3}$ & $1.90, \mathrm{~s}$ & $22.5, \mathrm{CH}_{3}$ \\
\hline 7-OMe & $3.85, \mathrm{~s}$ & $56.4, \mathrm{CH}_{3}$ & $3.95, \mathrm{~s}$ & $57.0, \mathrm{CH}_{3}$ \\
\hline
\end{tabular}

${ }^{\mathrm{a}}$ Measured at $600 \mathrm{MHz}$

${ }^{\mathrm{b}}$ Measured at $150 \mathrm{MHz}$

${ }^{\mathrm{c}}$ Measured at $800 \mathrm{MHz}$

${ }^{\mathrm{d}}$ Measured at $200 \mathrm{MHz}$

${ }^{\mathrm{e}}$ Detected by HMBC

\subsection{Plant Material}

The aerial parts of Piper mullesua Buch.-Ham. ex D. Don (Piperaceae) were collected from Mengyuan Village

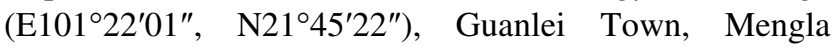
County, Xishuangbanna of Yunnan Province, People's Republic of China, in July 2014, and identified by one of the authors (C.-L.L.). A voucher specimen (No. 201401) was deposited at the Key Laboratory of Economic Plants and Biotechnology, Kunming Institute of Botany, Chinese Academy of Sciences.

\subsection{Extraction and Isolation}

The air-dried, powdered $P$. mullesua plant $(1.8 \mathrm{~kg})$ was exhaustively extracted with $\mathrm{MeOH}(4 \times 10 \mathrm{~L})$ at room temperature. The $\mathrm{MeOH}$ extracts $(92.5 \mathrm{~g})$ were suspended in $\mathrm{H}_{2} \mathrm{O}$ and further partitioned with petroleum ether and $\mathrm{CHCl}_{3}$. The petroleum ether-soluble part $(31.2 \mathrm{~g})$ and $\mathrm{CHCl}_{3}$-soluble part $(4.8 \mathrm{~g})$ were combined $(36.0 \mathrm{~g}$, part B) according to the testing results of thin-layer chromatography. The water phase was partitioned by D101 resin column chromatography to obtain the water-eluted part (discarded) and 95\% EtOH-eluted part (7.2 g, part A).
Table $3{ }^{1} \mathrm{H}$ and ${ }^{13} \mathrm{C}$ NMR Data of $\mathbf{3}$ and $\mathbf{3 a}$ in $\mathrm{CD}_{3} \mathrm{OD}$

\begin{tabular}{|c|c|c|c|c|}
\hline \multirow[t]{2}{*}{ No. } & \multicolumn{2}{|l|}{3} & \multicolumn{2}{|l|}{$\mathbf{3 a}$} \\
\hline & $\delta_{\mathrm{H}}(J \text { in } \mathrm{Hz})^{\mathrm{a}}$ & $\delta_{C}$, type $^{b}$ & $\delta_{\mathrm{H}}(J \text { in } \mathrm{Hz})^{\mathrm{c}}$ & $\delta_{\mathrm{C}}$, type $^{\mathrm{d}}$ \\
\hline 1 & & $173.0, \mathrm{C}$ & & $177.8, \mathrm{C}$ \\
\hline 3 & $3.62, \mathrm{t}(7.5)$ & $41.9, \mathrm{CH}_{2}$ & $3.80 \mathrm{dd} 7.67 .6$ & $42.1, \mathrm{CH}_{2}$ \\
\hline 4 & $2.95, \mathrm{~m}$ & $28.4, \mathrm{CH}_{2}$ & $3.13 \mathrm{dd} 7.67 .6$ & $27.1, \mathrm{CH}_{2}$ \\
\hline $4 a$ & & 141.1, C & & $140.4, \mathrm{C}$ \\
\hline 5 & $6.30, \mathrm{~s}$ & $117.8, \mathrm{CH}$ & $6.78, \mathrm{~s}$ & $113.5, \mathrm{CH}$ \\
\hline 6 & & 167.5, C & & $153.8^{\mathrm{e}}$ \\
\hline 7 & & $144.1, \mathrm{C}$ & & $140.8, \mathrm{C}$ \\
\hline 8 & & $145.9, \mathrm{C}$ & & $146.4, \mathrm{C}$ \\
\hline $8 \mathrm{a}$ & & $101.8, \mathrm{C}$ & & $107.9, \mathrm{C}$ \\
\hline 9 & 3.31 , overlapped & $34.7, \mathrm{CH}_{2}$ & $3.48 \mathrm{dd} 7.67 .6$ & $35.6, \mathrm{CH}_{2}$ \\
\hline 10 & $1.73, \mathrm{~m}$ & 27.3, $\mathrm{CH}_{2}$ & $1.75, \mathrm{~m}$ & 26.6, $\mathrm{CH}_{2}$ \\
\hline 11 & $1.63, \mathrm{~m}$ & $30.2, \mathrm{CH}_{2}$ & $1.65, \mathrm{~m}$ & $30.1, \mathrm{CH}_{2}$ \\
\hline 12 & $3.21, \mathrm{~m}$ & $40.0, \mathrm{CH}_{2}$ & $3.20 \mathrm{dd} 7.17 .1$ & $39.9, \mathrm{CH}_{2}$ \\
\hline $1^{\prime}$ & & 173.2, C & & 173.3, C \\
\hline $2^{\prime}$ & $1.90, \mathrm{~s}$ & 22.6, $\mathrm{CH}_{3}$ & $1.92, \mathrm{~s}$ & $22.6, \mathrm{CH}_{3}$ \\
\hline $1^{\prime \prime}$ & & $168.8, \mathrm{C}$ & & 171.3, C \\
\hline $2^{\prime \prime}$ & $2.98, \mathrm{~m}$ & $28.9, \mathrm{CH}_{2}$ & $3.06 \mathrm{dd} 7.47 .4$ & $28.9, \mathrm{CH}_{2}$ \\
\hline $3^{\prime \prime}$ & 1.90 , overlapped & $25.2, \mathrm{CH}_{2}$ & $1.94, \mathrm{~m}$ & $24.9, \mathrm{CH}_{2}$ \\
\hline $4^{\prime \prime}$ & $1.63, \mathrm{~m}$ & 29.7, $\mathrm{CH}_{2}$ & $1.65, \mathrm{~m}$ & $29.8, \mathrm{CH}_{2}$ \\
\hline $5^{\prime \prime}$ & $3.21, \mathrm{~m}$ & $40.1, \mathrm{CH}_{2}$ & $3.23 \mathrm{dd} 7.17 .1$ & $39.9, \mathrm{CH}_{2}$ \\
\hline $1^{\prime \prime \prime}$ & & 173.2, C & & 173.3, C \\
\hline $2^{\prime \prime \prime}$ & $1.93, \mathrm{~s}$ & 22.6, $\mathrm{CH}_{3}$ & $1.89, \mathrm{~s}$ & $22.6, \mathrm{CH}_{3}$ \\
\hline
\end{tabular}

${ }^{\mathrm{a}}$ Measured at $500 \mathrm{MHz}$

${ }^{\mathrm{b}}$ Measured at $125 \mathrm{MHz}$

${ }^{\mathrm{c}}$ Measured at $600 \mathrm{MHz}$

${ }^{\mathrm{d}}$ Measured at $150 \mathrm{MHz}$

${ }^{\mathrm{e}}$ Detected by HMBC

Part A was subjected to column chromatography (silica gel; $\left.\mathrm{CHCl}_{3} / \mathrm{MeOH}, 10: 1 \rightarrow 0: 1, \mathrm{v} / \mathrm{v}\right)$ to yield three fractions (A1-A3). Fraction A1 was separated on an RP-18 silica gel column eluted with $\mathrm{MeOH} / \mathrm{H}_{2} \mathrm{O}(10 \% \rightarrow 100 \%)$. The $40 \%$ $\mathrm{MeOH}$-eluted portion was purified by Sephadex LH-20 column chromatography $(\mathrm{MeOH})$ and semi-preparative HPLC (Aligent Zorbax SB-C ${ }_{18}$ column, $10 \times 250 \mathrm{~mm}$, $2 \mathrm{~mL} / \mathrm{min})$ to obtain $3\left(6.5 \mathrm{mg}, \mathrm{MeOH} / \mathrm{H}_{2} \mathrm{O}, 80: 20, t_{\mathrm{R}}=\right.$ $12.150 \mathrm{~min})$ and $11\left(6.9 \mathrm{mg}, \mathrm{MeCN} / \mathrm{H}_{2} \mathrm{O}, 15: 85, t_{\mathrm{R}}=\right.$ $5.190 \mathrm{~min})$.

Fraction A2 was separated on an RP-18 silica gel column eluted with $\mathrm{MeOH} / \mathrm{H}_{2} \mathrm{O}(10 \% \rightarrow 100 \%)$ to yield two main subfractions. The $5 \% \mathrm{MeOH}$-eluted portion was purified by Sephadex LH-20 column chromatography (MeOH) to yield two main subfractions (A2-1-1 and A2-12). Subfraction A2-1-1 was performed on preparative TLC $\left(\mathrm{CHCl}_{3} / \mathrm{MeOH}, 3: 1\right)$ to obtain $2(4.3 \mathrm{mg})$. Subfraction A21-2 was recrystallized from $\mathrm{MeOH}$ to obtain $\mathbf{1 0}(23.5 \mathrm{mg})$. 
Table $4{ }^{1} \mathrm{H}$ and ${ }^{13} \mathrm{C}$ NMR Data of 4-6 in $\mathrm{CD}_{3} \mathrm{OD}$

\begin{tabular}{|c|c|c|c|c|c|c|}
\hline \multirow[t]{2}{*}{ No. } & \multicolumn{2}{|l|}{4} & \multicolumn{2}{|l|}{5} & \multicolumn{2}{|l|}{6} \\
\hline & $\delta_{\mathrm{H}}(J \text { in } \mathrm{Hz})^{\mathrm{a}}$ & $\delta_{\mathrm{C}}$, type $^{b}$ & $\delta_{\mathrm{H}}(J \text { in } \mathrm{Hz})^{\mathrm{a}}$ & $\delta_{\mathrm{C}}$, type $^{b}$ & $\delta_{\mathrm{H}}(J \text { in } \mathrm{Hz})^{\mathrm{c}}$ & $\delta_{\mathrm{C}}$, type $^{\mathrm{d}}$ \\
\hline 1 & & $166.2, \mathrm{C}$ & & $164.9, \mathrm{C}$ & & 165.0, C \\
\hline 2 & $3.82, \mathrm{dd}(12.4,2.1)$ & $75.1, \mathrm{CH}$ & 3.78, br s & 79.1, CH & $3.68, \mathrm{~d}(2.5)$ & $80.6, \mathrm{CH}$ \\
\hline 3 & $\begin{array}{l}1.96, \mathrm{~m} \\
1.57, \mathrm{~m}\end{array}$ & $36.2, \mathrm{CH}_{2}$ & $2.11, \mathrm{~m}$ & $34.1, \mathrm{CH}$ & $2.42, \mathrm{~m}$ & $27.8, \mathrm{CH}$ \\
\hline 4 & $1.56, \mathrm{~m}$ & $25.9, \mathrm{CH}$ & $\begin{array}{l}1.61, \mathrm{~m} \\
1.51, \mathrm{~m}\end{array}$ & $30.6, \mathrm{CH}_{2}$ & $1.03, \mathrm{~d}(6.7)$ & $20.2, \mathrm{CH}_{3}$ \\
\hline 5 & $0.95, \mathrm{~d}(6.3)$ & $24.2, \mathrm{CH}_{3}$ & $1.01, \mathrm{dd}(7.3,7.3)$ & $12.2, \mathrm{CH}_{3}$ & $1.23, \mathrm{~d}(7.0)$ & 23.6, $\mathrm{CH}_{3}$ \\
\hline 6 & $0.98, \mathrm{~d}(5.9)$ & $21.5, \mathrm{CH}_{3}$ & $1.01, \mathrm{~d}(6.7)$ & 17.6, $\mathrm{CH}_{3}$ & & \\
\hline $1^{\prime}$ & & 140.0, C & & $139.9, \mathrm{C}$ & & 139.9, C \\
\hline $2^{\prime}, 6^{\prime}$ & $7.31, \mathrm{~d}(7.4)$ & $130.6, \mathrm{CH}$ & $7.29, \mathrm{~d}(7.4)$ & $130.8, \mathrm{CH}$ & $7.29, \mathrm{~d}(7.4)$ & $130.7, \mathrm{CH}$ \\
\hline $3^{\prime}, 5^{\prime}$ & $7.25, \mathrm{dd}(7.4,7.4)$ & $129.4, \mathrm{CH}$ & $7.23, \mathrm{dd}(7.4,7.4)$ & $129.2, \mathrm{CH}$ & $7.24, \mathrm{dd}(7.4,7.4)$ & $129.2, \mathrm{CH}$ \\
\hline $4^{\prime}$ & 7.17, dd $(7.4,7.4)$ & 127.6, CH & $7.15, \mathrm{dd}(7.4,7.4)$ & $127.4, \mathrm{CH}$ & $7.15, \mathrm{dd}(7.4,7.4)$ & $127.4, \mathrm{CH}$ \\
\hline $7^{\prime}$ & $\begin{array}{l}3.41, \text { dd }(14.1,4.4) \\
2.91, \text { dd }(14.1,10.7)\end{array}$ & $39.8, \mathrm{CH}_{2}$ & $\begin{array}{l}3.36, \mathrm{dd}(13.9,4.4) \\
2.86, \mathrm{dd}(13.9,10.2)\end{array}$ & $40.2, \mathrm{CH}_{2}$ & $\begin{array}{l}3.38, \mathrm{dd}(13.9,4.3) \\
2.85, \mathrm{dd}(13.9,10.3)\end{array}$ & $40.1, \mathrm{CH}_{2}$ \\
\hline $8^{\prime}$ & $4.71, \mathrm{dd}(10.7,4.4)$ & $57.6, \mathrm{CH}$ & $4.68, \mathrm{dd}(10.2,4.4)$ & $57.2, \mathrm{CH}$ & $4.70, \mathrm{dd}(10.3,4.3)$ & $57.1, \mathrm{CH}$ \\
\hline $9^{\prime}$ & & 177.2, C & & 177.2, C & & 177.2, C \\
\hline $\mathrm{NMe}$ & $2.85, \mathrm{~s}$ & $52.4, \mathrm{CH}_{3}$ & $2.98, \mathrm{~s}$ & $52.8, \mathrm{CH}_{3}$ & $2.96, \mathrm{~s}$ & $52.9, \mathrm{CH}_{3}$ \\
\hline
\end{tabular}

${ }^{\text {a}}$ Measured at $600 \mathrm{MHz}$

${ }^{\mathrm{b}}$ Measured at $150 \mathrm{MHz}$

${ }^{\mathrm{c}}$ Measured at $400 \mathrm{MHz}$

${ }^{\mathrm{d}}$ Measured at $100 \mathrm{MHz}$

The $30 \% \mathrm{MeOH}$-eluted portion was purified by Sephadex LH-20 column chromatography $(\mathrm{MeOH})$ and semipreparative HPLC [Welch Ultimate AQ- $\mathrm{C}_{18}$ column, $5.0 \mu \mathrm{m}, \phi$ $4.6 \times 300 \mathrm{~mm}, \quad \mathrm{MeCN} / \mathrm{H}_{2} \mathrm{O}$ (containing $0.05 \% \mathrm{TFA}$ ), 20:80, $1 \mathrm{~mL} / \mathrm{min}$ ] to obtain $\mathbf{1 3}\left(2.0 \mathrm{mg}, t_{\mathrm{R}}=5.796 \mathrm{~min}\right)$.

Fraction A3 was separated on an RP-18 silica gel column eluted with $\mathrm{MeOH} / \mathrm{H}_{2} \mathrm{O}(10 \% \rightarrow 100 \%)$ to yield two main subfractions. The $15 \% \mathrm{MeOH}$-eluted portion was purified by Sephadex LH-20 column chromatography $(\mathrm{MeOH})$ and semipreparative HPLC (Aligent Zorbax SB$\mathrm{C}_{18}$ column, $10 \times 250 \mathrm{~mm}, \mathrm{MeOH} / \mathrm{H}_{2} \mathrm{O}, 20: 80,2 \mathrm{~mL} / \mathrm{min}$ ) to obtain $7\left(4.5 \mathrm{mg}, t_{\mathrm{R}}=14.369 \mathrm{~min}\right), 6\left(8.0 \mathrm{mg}, t_{\mathrm{R}}=\right.$ $15.398 \mathrm{~min}), \mathbf{9}\left(24.0 \mathrm{mg}, t_{\mathrm{R}}=17.255 \mathrm{~min}\right), \mathbf{8}\left(38.4 \mathrm{mg}, t_{\mathrm{R}}=\right.$ $22.038 \mathrm{~min}), 5\left(27.3 \mathrm{mg}, t_{\mathrm{R}}=23.055 \mathrm{~min}\right)$, and $4(8.5 \mathrm{mg}$, $\left.t_{\mathrm{R}}=38.045 \mathrm{~min}\right)$. The $35 \% \mathrm{MeOH}$-eluted portion was purified by Sephadex LH-20 column chromatography $(\mathrm{MeOH})$ and semipreparative HPLC [Welch Ultimate AQ$\mathrm{C}_{18}$ column, $5.0 \mu \mathrm{m}, \phi 4.6 \times 300 \mathrm{~mm}, \mathrm{MeCN} / \mathrm{H}_{2} \mathrm{O}$ (containing $0.05 \%$ TFA), $20: 80,1 \mathrm{~mL} / \mathrm{min}]$ to obtain $\mathbf{1 2}$ (3.6 mg, $t_{\mathrm{R}}=9.300 \mathrm{~min}$ ).

Part B was subjected to column chromatography (silica gel; petroleum ether/acetone, 20:1 $\rightarrow 0: 1$, v/v) to yield five fractions (B1-B5). Fraction B1 was separated on an RP-18 silica gel column eluted with $\mathrm{MeOH} / \mathrm{H}_{2} \mathrm{O}(10 \% \rightarrow 100 \%)$ to yield four main subfractions. The $70 \% \mathrm{MeOH}$-eluted portion was purified by Sephadex LH-20 column chromatography $(\mathrm{MeOH})$ and silica gel column chromatography (petroleum ether/acetone, $40: 1, \mathrm{v} / \mathrm{v}$ ) to obtain $\mathbf{2 8}$ $(3.3 \mathrm{mg})$ and $29(4.9 \mathrm{mg})$. The $85 \% \mathrm{MeOH}$-eluted portion was purified by Sephadex LH-20 column chromatography $(\mathrm{MeOH})$ and preparative TLC (petroleum ether/EtOAc, 20:1, v/v) to obtain 32 (13.5 mg). The $90 \% \mathrm{MeOH}$-eluted portion was purified by Sephadex LH-20 column chromatography $(\mathrm{MeOH})$ to obtain $\mathbf{3 1}(2.3 \mathrm{mg})$ recrystallized from $\mathrm{MeOH}$. The $95 \% \mathrm{MeOH}$-eluted portion was purified by Sephadex LH-20 column chromatography $(\mathrm{MeOH})$ and semipreparative HPLC (Aligent Zorbax SB-C 18 column, $\left.10 \times 250 \mathrm{~mm}, \mathrm{MeOH} / \mathrm{H}_{2} \mathrm{O}, 100: 0,2 \mathrm{~mL} / \mathrm{min}\right)$ to obtain 34 $\left(6.0 \mathrm{mg}, t_{\mathrm{R}}=19.574 \mathrm{~min}\right)$ and $37\left(1.5 \mathrm{mg}, t_{\mathrm{R}}=26.868 \mathrm{~min}\right)$.

Fraction B2 was separated on an RP-18 silica gel column eluted with $\mathrm{MeOH} / \mathrm{H}_{2} \mathrm{O}(10 \% \rightarrow 100 \%)$ to yield two main subfractions. The $80 \% \mathrm{MeOH}$-eluted portion was purified by Sephadex LH-20 column chromatography $(\mathrm{MeOH})$ and semipreparative HPLC (Aligent Zorbax SB$\mathrm{C}_{18}$ column, $\left.10 \times 250 \mathrm{~mm}, 2 \mathrm{~mL} / \mathrm{min}\right)$ to obtain $\mathbf{1 4}$ ( $\left.86.7 \mathrm{mg}, \mathrm{MeOH} / \mathrm{H}_{2} \mathrm{O}, 80: 20, t_{\mathrm{R}}=16.778 \mathrm{~min}\right), \mathbf{2 6}(6.4 \mathrm{mg}$, $\left.\mathrm{MeOH} / \mathrm{H}_{2} \mathrm{O}, 75: 25, t_{\mathrm{R}}=8.558 \mathrm{~min}\right), 21(16.0 \mathrm{mg}, \mathrm{MeOH} /$ $\left.\mathrm{H}_{2} \mathrm{O}, 80: 20, t_{\mathrm{R}}=9.164 \mathrm{~min}\right)$, and $23\left(18.9 \mathrm{mg}, \mathrm{MeOH} / \mathrm{H}_{2} \mathrm{O}\right.$, 
Table $5{ }^{1} \mathrm{H}$ and ${ }^{13} \mathrm{C}$ NMR Data of 7-9 in $\mathrm{CD}_{3} \mathrm{OD}$

\begin{tabular}{|c|c|c|c|c|c|c|}
\hline \multirow[t]{2}{*}{ No. } & \multicolumn{2}{|l|}{7} & \multicolumn{2}{|l|}{8} & \multicolumn{2}{|l|}{9} \\
\hline & $\delta_{\mathrm{H}}(J \text { in } \mathrm{Hz})^{\mathrm{a}}$ & $\delta_{\mathrm{C}}$, type $^{\mathrm{b}}$ & $\delta_{\mathrm{H}}(J \text { in } \mathrm{Hz})^{\mathrm{a}}$ & $\delta_{\mathrm{C}}$, type $^{\mathrm{c}}$ & $\delta_{\mathrm{H}}(J \text { in } \mathrm{Hz})^{\mathrm{d}}$ & $\delta_{\mathrm{C}}$, type $^{\mathrm{c}}$ \\
\hline 1 & & 166.0, C & & $164.8, \mathrm{C}$ & & 164.9, C \\
\hline 2 & $3.71, \mathrm{dd}(11.8,1.8)$ & $75.1, \mathrm{CH}$ & 3.59 , br s & $79.3, \mathrm{CH}$ & $3.59, \mathrm{~d}(2.8)$ & $80.6, \mathrm{CH}$ \\
\hline 3 & $\begin{array}{l}1.93, \mathrm{~m} \\
1.54, \mathrm{~m}\end{array}$ & $36.1, \mathrm{CH}_{2}$ & $2.08, \mathrm{~m}$ & $34.1, \mathrm{CH}$ & $2.36, \mathrm{~m}$ & $27.8, \mathrm{CH}$ \\
\hline 4 & $1.54, \mathrm{~m}$ & $25.9, \mathrm{CH}$ & $\begin{array}{l}1.58, \mathrm{~m} \\
1.51, \mathrm{~m}\end{array}$ & $30.6, \mathrm{CH}_{2}$ & $1.01, \mathrm{~d}(6.7)$ & $20.2, \mathrm{CH}_{3}$ \\
\hline 5 & $0.93, \mathrm{~d}(6.0)$ & $24.1, \mathrm{CH}_{3}$ & 0.99 , overlapped & $12.1, \mathrm{CH}_{3}$ & $1.20, \mathrm{~d}(7.0)$ & $23.5, \mathrm{CH}_{3}$ \\
\hline 6 & $0.95, \mathrm{~d}(5.8)$ & $21.6, \mathrm{CH}_{3}$ & 0.99 , overlapped & $17.4, \mathrm{CH}_{3}$ & & \\
\hline $2^{\prime}$ & $7.15, \mathrm{~s}$ & $124.5, \mathrm{CH}$ & $7.13, \mathrm{~s}$ & $124.5, \mathrm{CH}$ & $7.14, \mathrm{~s}$ & $124.5, \mathrm{CH}$ \\
\hline $3^{\prime}$ & & $112.4, \mathrm{C}$ & & $112.4, \mathrm{C}$ & & $112.4, \mathrm{C}$ \\
\hline $3^{\prime} \mathrm{a}$ & & 129.1, C & & $129.4, \mathrm{C}$ & & $129.3, \mathrm{C}$ \\
\hline $4^{\prime}$ & 7.66 , br d (7.9) & $119.8, \mathrm{CH}$ & 7.66, br d (7.9) & $119.8, \mathrm{CH}$ & 7.67, br d (7.8) & $119.8, \mathrm{CH}$ \\
\hline $5^{\prime}$ & 7.01, ddd $(7.9,7.0,0.8)$ & 119.7, $\mathrm{CH}$ & 7.00 , ddd $(7.9,6.9,0.7)$ & 119.6, CH & 7.00, ddd $(7.8,7.5,1.0)$ & $119.6, \mathrm{CH}$ \\
\hline $6^{\prime}$ & 7.07 , ddd $(8.1,7.0,0.8)$ & $122.4, \mathrm{CH}$ & 7.06 , ddd $(8.1,6.9,0.7)$ & $122.2, \mathrm{CH}$ & 7.06 , ddd $(8.0,7.5,1.0)$ & $122.3, \mathrm{CH}$ \\
\hline $7^{\prime}$ & 7.30, br d (8.1) & $112.2, \mathrm{CH}$ & 7.28, br d $(8.1)$ & $112.1, \mathrm{CH}$ & 7.28, br d (8.0) & $112.1, \mathrm{CH}$ \\
\hline $7^{\prime} \mathrm{a}$ & & $137.8, \mathrm{C}$ & & $137.8, \mathrm{C}$ & & $137.8, \mathrm{C}$ \\
\hline $8^{\prime}$ & $\begin{array}{l}3.51, \mathrm{dd}(15.0,4.6) \\
3.15, \mathrm{dd}(15.0,9.9)\end{array}$ & $29.3, \mathrm{CH}_{2}$ & $\begin{array}{l}3.48 \text {, dd }(14.9,4.4) \\
3.11 \text {, dd }(14.9,9.5)\end{array}$ & $29.6, \mathrm{CH}_{2}$ & $\begin{array}{l}3.49, \text { dd }(14.8,4.3) \\
3.10, \text { dd }(14.8,9.8)\end{array}$ & 29.6, $\mathrm{CH}_{2}$ \\
\hline $9^{\prime}$ & $4.80, \mathrm{dd}(9.9,4.6)$ & $57.2, \mathrm{CH}$ & $4.75, \mathrm{dd}(9.5,4.4)$ & $56.9, \mathrm{CH}$ & 4.77, dd $(9.8,4.3)$ & $56.9, \mathrm{CH}$ \\
\hline $10^{\prime}$ & & $177.8, \mathrm{C}$ & & $177.9, \mathrm{C}$ & & $177.9, \mathrm{C}$ \\
\hline $\mathrm{NMe}$ & $2.69, \mathrm{~s}$ & $52.3, \mathrm{CH}_{3}$ & $2.84, \mathrm{~s}$ & $52.5, \mathrm{CH}_{3}$ & $2.80, \mathrm{~s}$ & $52.7, \mathrm{CH}_{3}$ \\
\hline
\end{tabular}

${ }^{\text {a }}$ Measured at $800 \mathrm{MHz}$

${ }^{b}$ Measured at $100 \mathrm{MHz}$

${ }^{\mathrm{c}}$ Measured at $125 \mathrm{MHz}$

${ }^{\mathrm{d}}$ Measured at $500 \mathrm{MHz}$

Table 6 The inhibitory effect of all compounds on aggregation of rabbit platelet induced by Thrombin (IIa) $(1 \mathrm{U} / \mathrm{mL})^{\mathrm{a}}$

\begin{tabular}{lll}
\hline Compound & Concentration $(\mu \mathrm{g} / \mathrm{mL})$ & Inhibition $(\%)^{\mathrm{b}}$ \\
\hline $\mathbf{2}$ & 300 & $19.0 \pm 9.6$ \\
$\mathbf{3}$ & 300 & $17.0 \pm 3.2$ \\
$\mathbf{5}$ & 300 & $11.5 \pm 7.9$ \\
$\mathbf{1 4}$ & 300 & $22.2 \pm 12.1$ \\
$\mathbf{2 7}$ & 300 & $21.9 \pm 11.3$ \\
$\mathbf{3 3}$ & 300 & $14.8 \pm 8.5$ \\
$\mathbf{3 4}$ & 300 & $12.8 \pm 9.4$ \\
Bivalirudin $^{\mathrm{c}}$ & 50 & $98.8 \pm 1.0$ \\
\hline
\end{tabular}

${ }^{\mathrm{a}}$ The inhibition of other tested compounds $(\mathbf{1}, \mathbf{4}, \mathbf{6}-\mathbf{1 3}, \mathbf{1 5}-\mathbf{2 6}, \mathbf{2 8}-\mathbf{3 2}$, and 35-37) was less than $10 \%$ at the concentration of $300 \mu \mathrm{g} / \mathrm{mL}$

${ }^{\mathrm{b}}$ Mean $\pm \mathrm{SD}, n=3$

${ }^{\mathrm{c}}$ Positive control

80:20, $\left.t_{\mathrm{R}}=19.593 \mathrm{~min}\right)$. The $90 \% \mathrm{MeOH}$-eluted portion was purified by Sephadex LH-20 column chromatography $(\mathrm{MeOH})$ and semipreparative HPLC (Aligent Zorbax SB$\mathrm{C}_{18}$ column, $10 \times 250 \mathrm{~mm}, \mathrm{MeCN} / \mathrm{H}_{2} \mathrm{O}, 99: 1,2 \mathrm{~mL} / \mathrm{min}$ ) to obtain $36\left(3.4 \mathrm{mg}, t_{\mathrm{R}}=37.378 \mathrm{~min}\right), 35\left(4.0 \mathrm{mg}, t_{\mathrm{R}}=\right.$ $42.351 \mathrm{~min})$, and $33\left(5.0 \mathrm{mg}, t_{\mathrm{R}}=45.179 \mathrm{~min}\right)$.

Fraction B3 was separated on an RP-18 silica gel column eluted with $\mathrm{MeOH} / \mathrm{H}_{2} \mathrm{O}(10 \% \rightarrow 100 \%)$ to yield two main subfractions. The $60 \% \mathrm{MeOH}-$ eluted portion was purified by Sephadex LH-20 column chromatography $(\mathrm{MeOH})$ to obtain 17 (32.6 $\mathrm{mg})$ and $\mathbf{2 4}(105.7 \mathrm{mg})$ recrystallized from $\mathrm{MeOH}$. The $70 \% \mathrm{MeOH}$-eluted portion was purified by Sephadex LH-20 column chromatography $(\mathrm{MeOH})$ and semipreparative HPLC (Aligent Zorbax SB$\mathrm{C}_{18}$ column, $\left.10 \times 250 \mathrm{~mm}, \mathrm{MeOH} / \mathrm{H}_{2} \mathrm{O}, 85: 15,2 \mathrm{~mL} / \mathrm{min}\right)$ to obtain 15 (10.3 $\left.\mathrm{mg}, t_{\mathrm{R}}=19.983 \mathrm{~min}\right)$.

Fraction B4 was separated on an RP-18 silica gel column eluted with $\mathrm{MeOH} / \mathrm{H}_{2} \mathrm{O}(10 \% \rightarrow 100 \%)$ to yield four main subfractions. The $60 \% \mathrm{MeOH}-$ eluted portion was purified by Sephadex LH-20 column chromatography $(\mathrm{MeOH})$ to obtain $1(169.9 \mathrm{mg})$ recrystallized from $\mathrm{MeOH}$. The $70 \%$ MeOH-eluted portion was isolated by column chromatography (Sephadex LH-20, MeOH; silica gel, petroleum ether/acetone, 30:1, v/v) and further purified by semipreparative HPLC (Agilent Zorbax SB-C ${ }_{18}$ column, 
Table 7 The inhibitory effect of all compounds on rabbit platelet aggregation induced by PAF $(0.4 \mu \mathrm{g} / \mathrm{mL})^{\mathrm{a}}$

\begin{tabular}{lll}
\hline Compound & Concentration $(\mu \mathrm{g} / \mathrm{mL})$ & Inhibition $(\%)^{\mathrm{b}}$ \\
\hline $\mathbf{1 1}^{\mathrm{c}}$ & 900 & $84.9 \pm 7.0$ \\
& 600 & $66.8 \pm 3.9$ \\
& 300 & $36.4 \pm 3.1$ \\
& 150 & $21.9 \pm 6.6$ \\
& 75 & $10.7 \pm 8.5$ \\
$\mathbf{1 4}^{\mathrm{c}}$ & 800 & $68.1 \pm 12.6$ \\
& 600 & $51.2 \pm 12.7$ \\
& 300 & $20.4 \pm 7.1$ \\
& 150 & $10.9 \pm 0.6$ \\
& 75 & $6.0 \pm 2.6$ \\
$\mathbf{1 5}^{\mathrm{c}}$ & 900 & $73.3 \pm 14.6$ \\
& 600 & $50.4 \pm 6.7$ \\
& 300 & $33.6 \pm 5.1$ \\
& 150 & $23.9 \pm 9.6$ \\
& 75 & $18.3 \pm 7.4$ \\
$\mathbf{2 0}$ & 300 & $16.8 \pm 6.7$ \\
$\mathbf{2 5}^{\text {Ginkgolide B }}$ & 300 & $17.0 \pm 7.6$ \\
& 25 & $90.2 \pm 10.4$ \\
& 12.5 & $56.9 \pm 11.2$ \\
& 6.25 & $33.9 \pm 9.3$ \\
& 3.125 & $19.5 \pm 9.6$ \\
& 1.5625 & $9.0 \pm 5.2$ \\
\hline
\end{tabular}

${ }^{a}$ The inhibition of other tested compounds (1-10, 12, 13, 16-19, 2124, and 26-37) was less than $10 \%$ at the concentration of $300 \mu \mathrm{g} / \mathrm{mL}$ ${ }^{\mathrm{b}}$ mean $\pm \mathrm{SD}, n=3$

${ }^{\mathrm{c}} \mathrm{The}_{\mathrm{IC}} \mathrm{C}_{50}$ values of $\mathbf{1 1}, \mathbf{1 4}$, and $\mathbf{1 5}$ was $470.3 \pm 51.6 \mu \mathrm{g} / \mathrm{mL}, 614.9 \pm$ $113.5 \mu \mathrm{g} / \mathrm{mL}$, and $579.7 \pm 139.1 \mu \mathrm{g} / \mathrm{mL}$, respectively

${ }^{\mathrm{d}}$ Positive control $\left(\mathrm{IC}_{50}=12.2 \pm 2.8 \mu \mathrm{g} / \mathrm{mL}\right)$

$9.4 \times 250 \mathrm{~mm}, 2 \mathrm{~mL} / \mathrm{min})$ to yield $20\left(8.5 \mathrm{mg}, \mathrm{MeOH} / \mathrm{H}_{2} \mathrm{O}\right.$, $\left.80: 20, t_{\mathrm{R}}=19.115 \mathrm{~min}\right), 25\left(3.0 \mathrm{mg}, \mathrm{MeOH}, 75: 25, t_{\mathrm{R}}=\right.$ $15.549 \mathrm{~min})$, and $18\left(16.0 \mathrm{mg} ; \mathrm{MeOH} / \mathrm{H}_{2} \mathrm{O}, 80: 20, t_{\mathrm{R}}=\right.$ $15.356 \mathrm{~min})$. The $80 \% \mathrm{MeOH}$-eluted portion was purified by Sephadex LH-20 column chromatography (MeOH) obtain 19 (2.0 mg) recrystallized from $\mathrm{MeOH}$. The $90 \%$ $\mathrm{MeOH} / \mathrm{H}_{2} \mathrm{O}$-eluted portion was purified by Sephadex LH20 column chromatography $(\mathrm{MeOH})$ and semipreparative HPLC (Aligent Zorbax SB-C 18 column, $10 \times 250 \mathrm{~mm}$, $\left.\mathrm{MeOH} / \mathrm{H}_{2} \mathrm{O}, 95: 5,2 \mathrm{~mL} / \mathrm{min}\right)$ to obtain $16\left(14.7 \mathrm{mg}, t_{\mathrm{R}}=\right.$ $14.172 \mathrm{~min})$.

Fraction B5 was separated on an RP-18 silica gel column eluted with $\mathrm{MeOH} / \mathrm{H}_{2} \mathrm{O}(10 \% \rightarrow 100 \%)$ to yield four main subfractions. The $10 \% \mathrm{MeOH}$-eluted portion was purified by column chromatography (Sephadex LH-20, $\mathrm{MeOH}$; preparative TLC, $\mathrm{CHCl}_{3} / \mathrm{MeOH}, 5: 1$, v/v) and semipreparative HPLC (Aligent Zorbax SB-C ${ }_{18}$ column, $10 \times 250 \mathrm{~mm}, \mathrm{MeOH} / \mathrm{H}_{2} \mathrm{O}, 40: 60,2 \mathrm{~mL} / \mathrm{min}$ ) to obtain 30
(18.2 $\left.\mathrm{mg}, t_{\mathrm{R}}=18.052 \mathrm{~min}\right)$. The $20 \% \mathrm{MeOH}$-eluted portion was purified by Sephadex LH-20 column chromatography $(\mathrm{MeOH})$ to obtain $27(52.3 \mathrm{mg})$ recrystallized from $\mathrm{MeOH}$. The $30 \%$ MeOH-eluted portion was purified by Sephadex LH-20 column chromatography $(\mathrm{MeOH})$ and semipreparative HPLC (Aligent Zorbax SB-C 18 column, $10 \times$ $250 \mathrm{~mm}, \mathrm{MeOH} / \mathrm{H}_{2} \mathrm{O}, 50: 50,2 \mathrm{~mL} / \mathrm{min}$ ) to obtain 22 $\left(18.0 \mathrm{mg}, t_{\mathrm{R}}=6.746 \mathrm{~min}\right)$.

\subsection{Spectroscopic Data of Compounds}

\subsubsection{Pipermullesine A (1)}

Pale yellow needles $\left(\mathrm{CHCl}_{3}\right)$; mp 90-93 ${ }^{\circ} \mathrm{C}$; UV $(\mathrm{MeOH})$ $\lambda_{\max }(\log \varepsilon) 334$ (4.22), 243 (4.11), 224 (3.94) nm; IR (KBr) $v_{\max } 1643,1595,1513,1461,1439,1415,1376,1345$, 1315, 1287, 1269, 1228, 1160, 1141, 1047, 1023, 907, $803 \mathrm{~cm}^{-1} ;{ }^{1} \mathrm{H}$ NMR and ${ }^{13} \mathrm{C}$ NMR data, see Table 1;

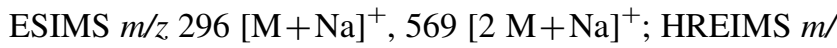
z $273.0997[\mathrm{M}]^{+}$(calcd for $\mathrm{C}_{15} \mathrm{H}_{15} \mathrm{NO}_{4}, 273.1001$ ).

Crystal data for pipermullesine A (1): $\mathrm{C}_{15} \mathrm{H}_{15} \mathrm{NO}_{4} \cdot \mathrm{H}_{2} \mathrm{O}$, $M=291.30$, monoclinic, $a=4.9028(8) \AA, b=21.159(3) \AA, c$ $=13.455(2) \AA, \alpha=90.00^{\circ}, \beta=92.120(2)^{\circ}, \gamma=90.00^{\circ}, \quad V=$ 1394.9(4) $\AA^{3}, T=100(2) \mathrm{K}$, space group $P 21 / n, Z=4$, $\mu(\operatorname{MoK} \alpha)=0.105 \mathrm{~mm}^{-1}, 14679$ reflections measured, and 3883 independent reflections $\left(R_{\text {int }}=0.0331\right)$. The final $R_{1}$ value was $0.0434(I>2 \sigma(I))$. The final $w R\left(F^{2}\right)$ value was $0.1212(I>2 \sigma(I))$. The final $R_{1}$ value was 0.0575 (all data). The final $w R\left(F^{2}\right)$ value was 0.1322 (all data). The goodness of fit on $F^{2}$ was 1.030. The crystallographic data for the structure of $\mathbf{1}$ have been deposited in the Cambridge Crystallographic Data Centre (deposition number CCDC 1529565). Copies of the data can be obtained free of charge from the CCDC via www.ccdc.cam.ac.uk.

\subsubsection{Pipermullesine B (2)}

Pale yellow powder; UV (MeOH) $\lambda_{\max }(\log \varepsilon) 402$ (3.62), 310 (3.07), 268 (3.43) nm; IR (KBr) $v_{\max } 3424,1622,1511$, 1466, 1441, 1368, 1354, 1235, 1208, 1180, $1039 \mathrm{~cm}^{-1} ;{ }^{1} \mathrm{H}$ and ${ }^{13} \mathrm{C}$ NMR data, see Table 2; ESIMS $m / z$ 291 $[\mathrm{M}+\mathrm{H}]^{+}$, $313[\mathrm{M}+\mathrm{Na}]^{+}$; HREIMS $\mathrm{m} / z 290.1620$ [M] $^{+}$(calcd for $\mathrm{C}_{16} \mathrm{H}_{22} \mathrm{~N}_{2} \mathrm{O}_{3}, 290.1630$ ).

\subsubsection{Pipermullesine C (3)}

Pale yellow powder; UV (MeOH) $\lambda_{\max }(\log \varepsilon) 400$ (3.62), 310 (3.10), 268 (3.42) nm; IR (KBr) $v_{\max }$ : 3376, 1721, 1630, 1607, 1590, 1562, 1511, 1462, 1438, 1384, 1337, 1271, 1237, 1211, 1101, 1076, $1037 \mathrm{~cm}^{-1} ;{ }^{1} \mathrm{H}$ and ${ }^{13} \mathrm{C}$ NMR data, see Table 3; ESIMS $m / z 415[\mathrm{M}+\mathrm{H}]^{+}, 437$ $[\mathrm{M}+\mathrm{Na}]^{+} ;$HREIMS $m / z \quad 414.2223 \quad[\mathrm{M}]^{+}$(calcd for $\mathrm{C}_{22} \mathrm{H}_{30} \mathrm{~N}_{4} \mathrm{O}_{4}, 414.2223$ ). 


\subsubsection{Pipermullamide A (4)}

White solid; $[\alpha]_{\mathrm{D}}^{25}-16.2$ ( $c$ 0.08, MeOH); UV (MeOH) $\lambda_{\max }$ $(\log \varepsilon) 204(3.35) \mathrm{nm} ; \mathrm{ECD} \Delta \varepsilon(c 0.08, \mathrm{MeOH})+1.35$ (217); IR (KBr) $v_{\max } 3429,1713,1626,1460,1415,1384$, 1299, 1274, 1126, 1079, $1046 \mathrm{~cm}^{-1} ;{ }^{1} \mathrm{H}$ and ${ }^{13} \mathrm{C}$ NMR data, see Table 4; ESIMS $m / z, 321[\mathrm{M}+\mathrm{H}]^{+}, 343[\mathrm{M}+\mathrm{Na}]^{+}$; HREIMS m/z $320.2102[\mathrm{M}]^{+}$(calcd for $\mathrm{C}_{18} \mathrm{H}_{28} \mathrm{~N}_{2} \mathrm{O}_{3}$, $320.2100)$.

\subsubsection{Pipermullamide B (5)}

White solid; $[\alpha]_{\mathrm{D}}^{19}-7.6(c 0.18, \mathrm{MeOH}) ; \mathrm{UV}(\mathrm{MeOH}) \lambda_{\max }$ $(\log \varepsilon) 203(4.00) \mathrm{nm}$; ECD $\Delta \varepsilon(c 0.012, \mathrm{MeOH})+1.48$ (214); IR (KBr) $v_{\max } 3442,1666,1609,1494,1456,1385$, $1312,1256,1226,1091,1031 \mathrm{~cm}^{-1} ;{ }^{1} \mathrm{H}$ and ${ }^{13} \mathrm{C}$ NMR data, see Table 4; ESIMS $m / z, 321[\mathrm{M}+\mathrm{H}]^{+}, 343[\mathrm{M}+\mathrm{Na}]^{+}$; HREIMS $\mathrm{m} / \mathrm{z} 320.2102[\mathrm{M}]^{+}$(calcd for $\mathrm{C}_{18} \mathrm{H}_{28} \mathrm{~N}_{2} \mathrm{O}_{3}$, 320.2100).

\subsubsection{Pipermullamide C (6)}

White solid; $[\alpha]_{\mathrm{D}}^{20}-33.1(c \quad 0.09, \mathrm{MeOH}) ; \mathrm{UV}(\mathrm{MeOH})$ $\lambda_{\max }(\log \varepsilon) 206(4.07) \mathrm{nm}$; ECD $\Delta \varepsilon(c 0.014, \mathrm{MeOH})+$ 2.50 (214); IR (KBr) $v_{\max } 3426,1673,1608,1494,1456$, 1383, 1315, 1257, 1225, 1096, $1032 \mathrm{~cm}^{-1} ;{ }^{1} \mathrm{H}$ and ${ }^{13} \mathrm{C}$ NMR data, see Table 4; ESIMS $\mathrm{m} / z 329[\mathrm{M}+\mathrm{Na}]^{+}$; HRESIMS $\quad m / z \quad 307.2017 \quad[\mathrm{M}+\mathrm{H}]^{+} \quad($ calcd for $\left.\mathrm{C}_{17} \mathrm{H}_{27} \mathrm{~N}_{2} \mathrm{O}_{3}, 307.2022\right)$.

\subsubsection{Pipermullamide D (7)}

White solid; $[\alpha]_{\mathrm{D}}^{21}-13.7$ ( $c$ 0.08, MeOH); UV (MeOH) $\lambda_{\max }$ (loge) 281 (3.47), 222 (4.28), 206 (4.16) nm; ECD $\Delta \varepsilon$ (c $0.037, \mathrm{MeOH})+1.40$ (233), -1.15 (222), -0.94 (214); IR (KBr) $v_{\max } 3426,1674,1611,1488,1459,1384,1259$, 1228, 1127, $1101 \mathrm{~cm}^{-1} ;{ }^{1} \mathrm{H}$ and ${ }^{13} \mathrm{C}$ NMR data, see Table 5; ESIMS $\mathrm{m} / \mathrm{z} 360[\mathrm{M}+\mathrm{H}]^{+}, 382[\mathrm{M}+\mathrm{Na}]^{+}$; HRESIMS $m / z 360.2288[\mathrm{M}+\mathrm{H}]^{+}$(calcd for $\mathrm{C}_{20} \mathrm{H}_{30} \mathrm{~N}_{3} \mathrm{O}_{3}$, 360.2287).

\subsubsection{Pipermullamide E (8)}

White solid; $[\alpha]_{\mathrm{D}}^{21}-32.2\left(\right.$ c 0.06, MeOH); UV (MeOH) $\lambda_{\max }$ (loge) 398 (1.74), 282 (3.09), 220 (3.90), 205 (3.89) nm; $\mathrm{ECD} \Delta \varepsilon(c 0.028, \mathrm{MeOH})+1.21(233),+1.87(225),-0.51$ (220), -3.34 (200); IR (KBr) $v_{\max } 3428,1681,1619,1452$, 1422, 1384, 1209, 1139, $1046 \mathrm{~cm}^{-1} ;{ }^{1} \mathrm{H}$ and ${ }^{13} \mathrm{C}$ NMR data, see Table 5; ESIMS $m / z, 360[\mathrm{M}+\mathrm{H}]^{+}, 382[\mathrm{M}+\mathrm{Na}]^{+}$; HRESIMS $m / z 360.2293[\mathrm{M}+\mathrm{H}]^{+}$(calcd for $\mathrm{C}_{20} \mathrm{H}_{30} \mathrm{~N}_{3} \mathrm{O}_{3}$, 360.2287).

\subsubsection{Pipermullamide F (9)}

White solid; $[\alpha]_{\mathrm{D}}^{20}-12.2(c 0.21, \mathrm{MeOH}) ; \mathrm{UV}(\mathrm{MeOH}) \lambda_{\max }$ (loge) 281 (3.53), 221 (4.31), 206 (4.15) nm; ECD $\Delta \varepsilon$ (c 0.013, MeOH) +1.30 (228), -1.80 (212), -4.48 (200); IR $(\mathrm{KBr}) v_{\max } 3415,3266,1672,1603,1491,1459,1384$, 1255, 1229, 1098, 961, $747 \mathrm{~cm}^{-1} ;{ }^{1} \mathrm{H}$ and ${ }^{13} \mathrm{C}$ NMR data, see Table 5; ESIMS $m / z 346[\mathrm{M}+\mathrm{H}]^{+}, 368[\mathrm{M}+\mathrm{Na}]^{+}$; HRESIMS $m / z, 346.2128[\mathrm{M}+\mathrm{H}]^{+}$(calcd for $\mathrm{C}_{19} \mathrm{H}_{28} \mathrm{~N}_{3} \mathrm{O}_{3}$, 346.2131).

\subsection{Preparation of Pipermullesine B Trifluoroacetate (2a)}

Compound 2 (1.6 mg, $0.00551 \mathrm{mmol}$ ) was performed on semipreparative HPLC [Welch Ultimate AQ- $\mathrm{C}_{18}$ column, $5.0 \mu \mathrm{m}, \phi 4.6 \times 300 \mathrm{~mm}, \mathrm{MeCN} / \mathrm{H}_{2} \mathrm{O}$ (containing $0.05 \%$ TFA), 20:80, $1.0 \mathrm{~mL} / \mathrm{min}$ ] to obtain $2 \mathrm{a}\left(2.0 \mathrm{mg}, t_{\mathrm{R}}=\right.$ $6.344 \mathrm{~min} ; 0.00516 \mathrm{mmol}, 94 \%$ yield): pale yellow needles $(\mathrm{MeOH}) ; \mathrm{mp} \quad 142-145{ }^{\circ} \mathrm{C} ;{ }^{1} \mathrm{H}$ and ${ }^{13} \mathrm{C}$ NMR data, see Table 2.

Crystal data for pipermullesine $\mathrm{B}$ trifluoroacetate (2a): $\mathrm{C}_{16} \mathrm{H}_{23} \mathrm{~N}_{2} \mathrm{O}_{3} \cdot \mathrm{C}_{2} \mathrm{~F}_{3} \mathrm{O}_{2}, \quad M=404.38, \quad a=7.6163(3) \AA ⿻$ 8.8383(3) $\AA, \quad c=14.8434(5) \AA, \quad \alpha=82.5370(10)^{\circ}, \quad \beta=$ $89.4170(10)^{\circ}, \gamma=74.5720(10)^{\circ}, V=954.71(6) \AA^{3}, T=100(2)$ $\mathrm{K}$, space group $P-1, Z=2, \mu(\mathrm{CuK} \alpha)=1.046 \mathrm{~mm}^{-1}, 13115$ reflections measured, 3381 independent reflections $\left(R_{i n t}=\right.$ $0.0623)$. The final $R_{l}$ value was $0.1027(I>2 \sigma(I))$. The final $w R\left(F^{2}\right)$ value was $0.2945(I>2 \sigma(I))$. The final $R_{l}$ value was 0.1048 (all data). The final $w R\left(F^{2}\right)$ value was 0.2990 (all data). The goodness of fit on $F^{2}$ was 1.412 . The crystallographic data for the structure of 2a have been deposited in the Cambridge Crystallographic Data Centre (deposition number CCDC 1529558). Copies of the data can be obtained free of charge from the CCDC via www.ccdc. cam.ac.uk.

\subsection{Preparation of Pipermullesine C Trifluoroacetate (3a)}

Compound 3 (4.0 mg, $0.00965 \mathrm{mmol}$ ) was performed on semipreparative HPLC [Welch Ultimate AQ- $\mathrm{C}_{18}$ column, $5.0 \mu \mathrm{m}, \phi 4.6 \times 300 \mathrm{~mm}, \mathrm{MeCN} / \mathrm{H}_{2} \mathrm{O}$ (containing $0.05 \%$ TFA), 20:80, $1.0 \mathrm{~mL} / \mathrm{min}$ ] to obtain $3 \mathrm{a}\left(4.5 \mathrm{mg}, t_{\mathrm{R}}=\right.$ $7.460 \mathrm{~min} ; 0.00880 \mathrm{mmol}, 91 \%$ yield): pale yellow needles (MeOH); mp $157-159{ }^{\circ} \mathrm{C}$; ${ }^{1} \mathrm{H}$ and ${ }^{13} \mathrm{C}$ NMR data, see Table 3.

Crystal data for pipermullesine $\mathrm{C}$ trifluoroacetate (3a): $\mathrm{C}_{22} \mathrm{H}_{31} \mathrm{~N}_{4} \mathrm{O}_{4} \cdot \mathrm{C}_{2} \mathrm{~F}_{3} \mathrm{O}_{2}, \quad M=528.53, a=8.5097(10) \AA, \quad b=$ $12.3631(15) \AA, \quad c=12.8583(15) \AA, \alpha=68.354(2)^{\circ}, \beta=$ 78.084(2) ${ }^{\circ}, \gamma=80.814(2)^{\circ}, V=1225.1(3) \AA^{3}, T=100(2) \mathrm{K}$, space group $P-1, Z=2, \mu(\mathrm{MoK} \alpha)=0.118 \mathrm{~mm}^{-1}, 13522$ 
reflections measured, 6727 independent reflections $\left(R_{\text {int }}=\right.$ $0.0383)$. The final $R_{l}$ values were $0.0528(I>2 \sigma(I))$. The final $w R\left(F^{2}\right)$ value was $0.1229(I>2 \sigma(I))$. The final $R_{l}$ value was 0.0879 (all data). The final $w R\left(F^{2}\right)$ value was 0.1414 (all data). The goodness of fit on $F^{2}$ was 1.020 . The crystallographic data for the structure of $\mathbf{3 a}$ have been deposited in the Cambridge Crystallographic Data Centre (deposition number CCDC 1589949). Copies of the data can be obtained free of charge from the CCDC via www. ccdc.cam.ac.uk.

\subsection{In vitro Platelet Aggregation Assay}

The inhibitory effects of compounds against rabbit platelet aggregation induced by PAF or Thrombin (IIa) were evaluated according to the published methods [50-53]. The details were presented in Supplementary Material.

\section{Conclusion}

Thirty-seven compounds were isolated from the folk Chinese medicine Piper mullesua with the "Huayu" function associated with the antiplatelet therapies. The antiplatelet compounds, especially (-)-mangochinine, pellitorine, and (2E,4E)- $N$-isobutyl-2,4-dodecadienamide, might be scientific evidence to support the traditional use of the plant as folk medicine. In order to make better use of the folk medicine to serve for human health, further research needs to be conducted on bioguided isolation of compounds from the plant, based on both in vitro and in vivo bioassay testing.

Acknowledgements This work was funded by the Southeast Asia Biodiversity Research Institute, Chinese Academy of Sciences (Y4ZK111B01), the Natural Science Foundation of Yunnan Province, China (2011FZ205), the International Partneship Program of Chinese Academy of Sciences (153631KYSB20160004), the Key Laboratory of Ethnomedicine (Minzu University of China) of Ministry of Education of China (KLEM-ZZ201806), and the National Natural Science Foundation of China (31761143001 \& 31161140345).

\section{Compliance with Ethical Standards}

Conflict of interest Authors declare that there is no conflict of interest associated with this work.

Open Access This article is distributed under the terms of the Creative Commons Attribution 4.0 International License (http://creative commons.org/licenses/by/4.0/), which permits unrestricted use, distribution, and reproduction in any medium, provided you give appropriate credit to the original author(s) and the source, provide a link to the Creative Commons license, and indicate if changes were made.

\section{References}

1. C. Chen, F.Q. Wang, X. Wang, Z.N. Xia, H. Guang, J. Tradit. Chin. Med. 37, 64-75 (2017)

2. X.L. Su, W. Su, Y. Wang, X. Ming, Y. Kong, Acta Pharm. Sinica 37, 1208-1217 (2016)

3. J.X. Zhuo, Y.H. Wang, X.L. Su, R.Q. Mei, J. Yang, Y. Kong, C. L. Long, Nat. Prod. Bioprospect. 6, 161-166 (2016)

4. Y.H. Wang, S.L. Morris Natschke, J. Yang, H.M. Niu, C.L. Long, K.H. Lee, J. Tradit. Complement. Med. 4, 8-16 (2014)

5. Editorial Board of "Zhonghua Bencao", Zhonghua Bencao, vol. 3 (Shanghai Scientific and Technological Press, Shanghai, 1999), pp. 424-449

6. K. Zhang, W. Ni, C.C. Chen, Y.T. Liu, Plant Divers. 20, 374-376 (1998)

7. Z.Q. Shen, Z.H. Chen, D.C. Wang, J. Kunming Med. Univ. 29, 23-25 (1997)

8. S. Srivastava, M.M. Gupta, V. Prajapati, A.K. Tripathi, S. Kumar, Phytother. Res. 15, 70-72 (2001)

9. S. Srivastava, M. Gupta, V. Prajapati, A. Tripathi, S. Kumar, Pharm. Biol. 39, 226-229 (2001)

10. S. Srivastava, M.M. Gupta, A.K. Tripathi, S. Kumar, Indian J. Chem. 39, 946-949 (2001)

11. S. Srivastava, R.K. Verma, M.M. Gupta, S. Kumar, J. Indian Chem. Soc. 77, 305-306 (2000)

12. D.D. Ding, Y.H. Wang, Y.H. Chen, R.Q. Mei, J. Yang, J.F. Luo, Y. Li, C.L. Long, Y. Kong, Phytochemistry 129, 36-44 (2016)

13. D.D. Zhang, J. Yang, J.F. Luo, X.N. Li, C.L. Long, Y.H. Wang, J. Asian Nat. Prod. Res. (2017). https://doi.org/10.1080/10286020. 10282017.11346630

14. E. Claveau, I. Gillaizeau, J. Blu, A. Bruel, G. Coudert, J. Org. Chem. 72, 4832-4836 (2007)

15. S.V. Eswaran, D. Kaur, K. Khamaru, S. Prabhakar, T. Sony, P. Raghunathan, B. Ganguly, Tetrahedron Lett. 57, 1899-1902 (2016)

16. I.I. Rodríguez, A.D. Rodríguez, J. Nat. Prod. 66, 855-857 (2003)

17. C.J. Li, D. Brownson, T.J. Mabry, C. Perera, E.A. Bell, Phytochemistry 42, 443-445 (1996)

18. Y.H. Wang, C.L. Long, F.M. Yang, X. Wang, Q.Y. Sun, H.S. Wang, Y.N. Shi, G.H. Tang, J. Nat. Prod. 72, 1151-1154 (2009)

19. M. Gacek, K. Undheim, Tetrahedron 29, 863-866 (1973)

20. S.X. Qiu, C. Liu, S.X. Zhao, Z.C. Xia, N.R. Farnsworth, H.H.S. Fong, Tetrahedron Lett. 29, 4167-4170 (1998)

21. Y. Nishiyama, M. Moriyasu, M. Ichimaru, K. Iwasa, A. Kato, S. G. Mathenge, P.B. Chalo Mutiso, F.D. Juma, Phytochemistry 65, 939-944 (2004)

22. A. Kato, M. Moriyasu, M. Ichimaru, Y. Nishiyama, F.D. Juma, J. N. Nganga, S.G. Mathenge, J.O. Ogeto, Phytochem. Anal. 6, 8995 (1995)

23. I.K. Park, S.G. Lee, S.C. Shin, J.D. Park, Y.J. Ahn, J. Agric. Food Chem. 50, 1866-1870 (2002)

24. J.R. Stöhr, P.G. Xiao, R. Bauer, Planta Med. 65, 175-177 (1999)

25. A. Banerji, C. Das, Phytochemistry 28, 3039-3042 (1989)

26. A. Banerji, D. Bandyopadhyay, M. Sarkar, A.K. Siddhanta, S.C. Pal, S. Ghosh, K. Abraham, J.N. Shoolery, Phytochemistry 24, 279-284 (1985)

27. K. Likhitwitayawuid, N. Ruangrungsi, G.L. Lange, C.P. Decicco, Tetrahedron 43, 3689-3694 (1987)

28. V.S. Parmar, S.C. Jain, S. Gupta, S. Talwar, V.K. Rajwanshi, R. Kumar, A. Azim, S. Malhotra, N. Kumar, R. Jain, Phytochemistry 49, 1069-1078 (1998) 
29. E. Nomura, A. Kashiwada, A. Hosoda, K. Nakamura, H. Morishita, T. Tsuno, H. Taniguchi, Bioorgan. Med. Chem. 11, 38073813 (2003)

30. D. Takaoka, K. Watanabe, M. Hiroi, Bull. Chem. Soc. Jpn 49, 3564-3566 (1976)

31. T. Zhuang, B. Xu, L. Huang, X. Chen, J. Liang, W. Qu, J. China. Pharm. Univ. 45, 410-412 (2014)

32. H. Shimomura, Y. Sashida, M. Oohara, Phytochemistry 27, 634636 (1988)

33. D.D. de L. Moreira, E.F. Guimaraes, M.A.C. Kaplan, Phytochemistry 55, 783-786 (2000)

34. S.H. Lee, I.K. Dong, J.A. Kim, Y. Jahng, Heterocycl. Commun. 11, 407-410 (2005)

35. K. Obst, B. Lieder, K.V. Reichelt, M. Backes, S. Paetz, K. Geißler, G. Krammer, V. Somoza, J.P. Ley, K.H. Engel, Phytochemistry 135, 181-190 (2017)

36. G. Erosa-Rejón, L.M. Peña-Rodríguez, O. Sterner, J. Mex. Chem. Soc. 53, 44-47 (2009)

37. T.V. Sung, B. Steffan, W. Steglich, G. Klebe, G. Adam, Phytochemistry 31, 1659-1661 (1992)

38. J.J. Sims, J.A. Pettus Jr., Phytochemistry 15, 1076-1077 (1976)

39. J.D.P. Teresa, J.G. Urones, I.S. Marcos, J.F. Ferreras, A.L. Bertelloni, P.B. Barcala, Phytochemistry 26, 1481-1485 (1987)

40. M. Matsuo, S. Urano, Tetrahedron 32, 229-231 (1976)

41. W.H. Chen, G.Y. Chen, J. Wang, Y. Hui, L. Liu, J.J. Han, X.P. Song, Chem. Nat. Compd. 51, 797-799 (2015)
42. J.G. Cui, L.M. Zeng, J.Y. Su, C.W. Lin, Chem. Res. Chin. Univ. 18, 400-404 (2002)

43. E.S. Elkhayat, S.R. Ibrahim, G.A. Mohamed, S.A. Ross, Nat. Prod. Res. 30, 814-820 (2016)

44. M.Y. Xia, L. Wang, Y.H. Wang, Nat. Prod. Res. Dev. 28, 16761685 (2016)

45. C.Y. Li, W.J. Tsai, A.G. Damu, E.J. Lee, T.S. Wu, N.X. Dung, T. D. Thang, L. Thanh, J. Agric. Food Chem. 55, 9436-9442 (2007)

46. T. Rukachaisirikul, P. Siriwattanakit, K. Sukcharoenphol, C. Wongvein, P. Ruttanaweang, P. Wongwattanavuch, A. Suksamrarn, J. Ethnopharmacol. 93, 173-176 (2004)

47. Y.N. Shi, F.F. Liu, M.R. Jacob, X.C. Li, H.T. Zhu, D. Wang, R.R. Cheng, C.R. Yang, M. Xu, Y.J. Zhang, Planta Med. 83, 143-150 (2016)

48. S.V. Pullela, A.K. Tiwari, U.S. Vanka, A. Vummenthula, H.B. Tatipaka, K.R. Dasari, I.A. Khan, M.R. Janaswamy, J. Ethnopharmacol. 108, 445-449 (2006)

49. S.K. Ku, I.C. Lee, J.A. Kim, J.S. Bae, Fitoterapia 91, 1-8 (2013)

50. G.V. Born, Nature 194, 927-929 (1962)

51. L.J. Küster, J. Filep, J.C. Frölich, Thromb. Res. 43, 425 (1986)

52. M. Wu, D. Wen, N. Gao, C. Xiao, L. Yang, L. Xu, W. Lian, W. Peng, J. Jiang, J. Zhao, Eur. J. Med. Chem. 92, 257-269 (2015)

53. X.Y. Zhu, H.C. Liu, S.Y. Guo, B. Xia, R.S. Song, Q.C. Lao, Y.X. Xuan, C.Q. Li, Zebrafish 13, 335-344 (2016) 